\title{
On the Meta Distribution of Coverage Probability in Uplink Cellular Networks
}

\author{
Hesham ElSawy, Senior Member, IEEE and Mohamed-Slim Alouini, Fellow, IEEE
}

\begin{abstract}
This letter studies the meta distribution of coverage probability (CP), within a stochastic geometry framework, for cellular uplink transmission with fractional path-loss inversion power control. Using the widely accepted Poisson point process (PPP) for modeling the spatial locations of base stations (BSs), we obtain the percentiles of users that achieve a target uplink CP over an arbitrary, but fixed, realization of the PPP. To this end, the effect of the users activity factor $(p)$ and the path-loss compensation factor $(\epsilon)$ on the uplink performance are analyzed. The results show that decreasing $p$ and/or increasing $\epsilon$ reduce the $C P$ variation around the spatially averaged value.
\end{abstract}

Index Terms-Stochastic geometry, meta distribution, uplink cellular networks, fractional channel inversion power control.

\section{INTRODUCTION}

Mathematical models based on Stochastic geometry have been widely utilized to analyze, understand, and obtain design insights for cellular networks [1]. However, most of the stochastic geometry models are confined to spatial averages of performance metrics (e.g., coverage probability [CP]) [1]. Such spatial averages fuse the fast fading and slow spatial topology variations despite their different time scales. Furthermore, spatial averages do not reveal information about percentiles of users that achieve certain performance measures, which is a fundamental design objective for cellular operators to identity the network quality of service (QoS).

The distribution of the signal-to-interference-ratio (SIR), or equivalently the $\mathrm{CP}$, for a fixed, yet arbitrary, realization of the network provides a fairly general and insightful characterization for cellular networks. Such distribution is known in the literature as the meta distribution [2], which is used to reveal interesting insights about the network performance such as i) the discrepancies among the users performance; and ii) the percentage of users experiencing a certain QoS. ${ }^{1}$ Since an exact expression for the meta distribution is generally hard to obtain, statistical inequalities (e.g., Markov, Chebyshev, and Chernoff) are usually sought [2], [5]. Such inequalities require the moments of the $\mathrm{CP}$, which are easier to obtain. The moments of the $\mathrm{CP}$ can also be used to express the meta distribution in an integral form or for approximating the meta distribution via moment matching [2].

The authors are with King Abdullah University of Science and Technology (KAUST), Thuwal, Makkah Province, Saudi Arabia, email: \{hesham.elsawy, slim.alouini\}@kaust.edu.sa.

This research was supported by the Sensor Research Initiative through the Office of Sponsored Research at the King Abdullah University of Science and Technology, Thuwal, Makkah Province, Saudi Arabia.

${ }^{1}$ The meta distribution is different form the rate coverage that is a function of the spatially averaged CP [3], [4].
This letter derives the meta distribution of the $\mathrm{CP}$ in uplink cellular networks. According to the 3GPP standard [6], we consider fractional path-loss inversion power control (PC). Assuming a Poisson cellular network, integral expressions for all moments, that reduce to closed forms for full pathloss inversion, of the CP are obtained. The meta distribution for the $\mathrm{CP}$ is then approximated via the beta distribution using moment matching. The results reveal the significant discrepancies in users percentiles performance for different path-loss compensation factors that show equivalent spatially averaged CP. Interestingly, decreasing the activity factor $p$ not only improves the spatially averaged $\mathrm{CP}$, but also reduces the discrepancies of the $\mathrm{CP}$ among simultaneously active users.

\section{System Model}

This letter focuses on the uplink transmission in a single tier cellular network where the BSs are distributed according to a PPP, denoted by $\Psi \in \mathbb{R}^{2}$, with intensity $\lambda_{b} \mathrm{BS} / \mathrm{km}^{2}$. The users (UEs) point process, denoted by $u_{i} \in \Phi$, is generated by uniformly and independently dropping one UE in the Voronoi cell of each BSs $b_{i} \in \Psi$. In each time slot, each UE becomes active independently with probability $p$. When a UE is active, it transmits with a fractional path-loss inversion PC with compensation factor $\epsilon$. For simplicity, we utilize the commonly used power-law path-loss model with exponent $\eta>2$. Hence, the transmit power of a UE located $r$ meters away from its serving BS is given by $\rho r^{\eta \epsilon}$, where $\rho$ is a PC parameter to adjust the received power at the serving BS. A Rayleigh fading environment, with unit mean channel power gains, is assumed. All channel gains are assumed to be independent and identically distributed. We assume that the BSs are dense enough such that all UEs can invert their path-loss toward their serving BSs almost surely.

\section{ANALYSIS}

For an arbitrary, but fixed, realization of $\Psi$ and $\Phi$, let $u_{\circ} \in \Phi$ and $b_{\circ}=\operatorname{argmin}_{b \in \Psi}\left\|u_{\circ}-b\right\|$, where $\|\cdot\|$ denotes the Euclidean norm, be a randomly selected UE and its serving BS. Ignoring the thermal noise, the $\mathrm{CP}$ for the selected test link is

$$
\begin{aligned}
P_{s}(\theta) & =\mathbb{P}^{!}\left\{\frac{P_{\circ} h_{\circ}\left\|u_{\circ}-b_{\circ}\right\|^{-\eta}}{\sum_{u_{i} \in \Phi \backslash u_{\circ}} a_{i} P_{i} h_{i}\left\|u_{i}-b_{\circ}\right\|^{-\eta}}>\theta \mid \Psi, \Phi\right\} \\
& =\prod_{r_{i} \in \Phi_{\circ}}\left(\frac{p}{1+\frac{\theta P_{i} r_{\circ}^{\eta(1-\epsilon)}}{\rho r_{i}^{\eta}}}+1-p\right),
\end{aligned}
$$


where $\mathbb{P} !\{\cdot\}$ is the reduced Palm probability, $P_{\circ}$ is the transmission power of the test UE, $h_{\circ} \sim \exp (1)$ is the intended channel power gain, $r_{\circ}=\left\|u_{\circ}-b_{\circ}\right\|$ is the test link distance, $a_{i} \sim \operatorname{Bernoulli}(p)$ denotes the $i^{t h}$ interfering UE activity, $P_{i}$ is the transmission power of the $i^{\text {th }}$ interfering UE, $h_{i} \sim \exp (1)$ is the $i^{t h}$ interfering channel power gain, and $\Phi_{\circ}=\left\{\left\|b_{\circ}-\left\{\Phi \backslash u_{\circ}\right\}\right\|\right\}$ is the set containing the distances between interfering UEs and the test BS. In the analysis, the Palm probability in (1) is captured via the intensity function of $\Phi_{\circ}$ and the probability density function (pdf) of $P_{i}$, which describe the panorama of interferers as seen from the test BS perspective [3], [4], [7], [8]. The result in (1) is obtained by the substitution $P_{\circ}=\rho r_{\circ}^{\eta \epsilon}$ and averaging over the channel gains and the UEs activity (i.e., $a_{i}$ ) (cf. [2, Appendix A]). Note that $P_{i}$ is not averaged out in (1) because it does not take different realizations over time as $\Psi$ and $\Phi$ are fixed.

From the spatial domain perspective, the $\mathrm{CP}$ is a random variable that changes from one link to another in the realization of $\Psi \cup \Phi$. To capture such randomness, [2] defines the meta distribution for the $\mathrm{CP}$ as

$$
\bar{F}(\theta, \alpha)=\mathbb{P}^{!}\left\{P_{s}(\theta)>\alpha \mid \Psi, \Phi\right\},
$$

which is a two parameter complementary cumulative distribution function (ccdf) that defines the percentiles of users $(\alpha)$ that achieves a certain $P_{s}(\theta)$ when conditioned on a realization for $\Psi \cup \Phi$. Despite the fact that $\Psi$ and $\Phi$ are fixed, the randomness in (2) originates from the different BS and UE separations within $\Psi \cup \Phi$ along with the different panorama of interfering UEs seen by each BS $b \in \Psi$. The $b^{t h}$ moment of the CP across all links in an arbitrary fixed realization of $\Psi \cup \Phi$ is given by

$$
M_{b}=\mathbb{E}^{!}\left[\prod_{r_{i} \in \Phi_{\circ}}\left(\frac{p}{1+\frac{\theta P_{i} r_{\rho}^{\eta(1-\epsilon)}}{\rho r_{i}^{\eta}}}+1-p\right)^{b}\right] .
$$

The averaging in (3) is over the intended link distances $r_{\mathrm{o}}$, the interfering link distances $r_{i}$ and the transmission powers $P_{i}$. It is worth noting that the uplink scenario has two fundamental differences from the downlink scenario analyzed in [2]. First, the association does not impose a spatial interference protection in the uplink [3], [4], [7]. Hence, an interfering UE can be arbitrary close to a BS than its intended UE (i.e., $r_{i}$ can be less than $r_{\mathrm{o}}$ ). Second, the transmission powers of the interfering UEs are random due to the employed fractional channel inversion PC.

The distribution of the transmission powers of the UEs in $\Phi_{\circ}$ is given by the following lemma.

Lemma 1. The distribution of the transmission power $P_{i}$ of the $i^{\text {th }} U E$ that is located at the distance $r_{i} \in \Phi_{\circ}$ from the test $B S$ is given by

$$
f_{P_{i}}\left(z \mid r_{i}\right)=\frac{2 \pi \lambda_{b} z^{\frac{2}{\eta \epsilon}-1} e^{-\pi \lambda_{b}\left(\frac{z}{\rho}\right)^{\frac{2}{\eta \epsilon}}}}{\eta \epsilon \rho^{\frac{2}{\eta \epsilon}-1}\left(1-e^{-\pi \lambda_{b} r_{i}^{2}}\right)} ; \quad 0 \leq z \leq \rho r_{i}^{\eta \epsilon} .
$$

Proof: See [7, Lemmal].

Despite that $\Psi$ is a PPP, the UEs point process $\Phi$ is not a PPP [3], [4], [7], [8]. ${ }^{2}$ Furthermore, the transmission powers of

\footnotetext{
${ }^{2} \mathrm{~A}$ comprehensive study for the user point process is available in [9].
}

nearby UEs are correlated due to the correlation of the sizes of adjacent cells. For tractable analysis, the transmission power correlations are ignored and the interference seen from $\left\{\Phi \backslash u_{\circ}\right\}$ at $b_{o}$ is approximated with the interference seen from a PPP $\tilde{\Phi} \in \mathbb{R}^{2}$ with intensity function $\lambda(x)=\left(1-e^{-\pi \lambda_{b}\|x\|^{2}}\right) \lambda_{b}$. Such approximations are shown to be accurate in [3], [4], [8] for the spatially averaged $P_{s}(\theta)$ and are validated in this letter for the meta distribution $\bar{F}(\theta, \alpha)$. For the sake of mathematical convenience, we first use the following lemma to average out the transmission powers of the interfering UEs and to facilitate the subsequent stochastic geometry analysis.

Lemma 2. Let $\tilde{\Phi}_{\circ}=\left\{w_{i}=\frac{\left\|x_{i}\right\|^{\eta}}{P_{i}}, \forall\left\|x_{i}\right\| \in \tilde{\Phi}\right\}$, where $\tilde{\Phi} \in$ $\mathbb{R}^{2}$ a PPP with intensity function $\lambda(x)=\left(1-e^{-\pi \lambda_{b}\|x\|^{2}}\right) \lambda_{b}$ that approximates the interference from $\left\{\Phi \backslash u_{\circ}\right\}$ at $b_{o}$. Then the moments in (3) can be approximated by

$$
M_{b} \approx \tilde{M}_{b}=\mathbb{E}^{!}\left[\prod_{w_{i} \in \tilde{\Phi}_{\circ}}\left(\frac{p}{1+\frac{\theta r_{o}^{\eta(1-\epsilon)}}{\rho w_{i}}}+1-p\right)^{b}\right],
$$

where $\tilde{\Phi}_{\circ} \in \mathbb{R}^{+}$is a PPP with the intensity function

$$
\tilde{\lambda}(w)=\frac{2(\pi \lambda)^{1-\epsilon} \rho^{\frac{2}{\eta}}}{\eta w^{1-\frac{2}{\eta}}} \gamma\left(1+\epsilon, \pi \lambda(\rho w)^{\frac{2}{\eta(1-\epsilon)}}\right),
$$

where $\gamma(a, b)=\int_{0}^{b} t^{a-1} e^{-t} \mathrm{~d} t$ is the lower incomplete gamma function.

Proof: First, we note that the approximation in (5) is due to approximating the interference from $\left\{\Phi \backslash u_{\circ}\right\}$ with the interference from $\tilde{\Phi}$. Otherwise, the results in the lemma are exact. The lemma is obtained by virtue of the Displacement and Mapping Theorems for the PPPs [10, Theorems $2.33 \& 2.34]$, in which the transformation $\tilde{\Phi}_{\circ}=\left\{w_{i}=\frac{\left\|x_{i}\right\|^{\eta}}{P_{i}}, \forall x_{i} \in \tilde{\Phi}\right\}$ is applied to obtain the unit power interfering UEs and the linear path-loss model shown in (5). The intensity in (6) is obtained in two steps as follows. The first step is by applying the Mapping theorem with the mapping function $r=\|x\|^{\eta}$, the intensity function becomes $\lambda(r)=\frac{2 \pi \lambda_{b} r^{\frac{2}{\eta}-1}}{\eta}\left(1-e^{-\pi \lambda_{b} r^{\frac{2}{\eta}}}\right)$. The second step is by applying the distance dependent random displacement with the PDF in (4), which gives the following displacement kernel

$$
\zeta(r, w)=\frac{2 \pi \lambda_{b} r^{\frac{2}{\eta \epsilon}} e^{-\pi \lambda_{b}\left(\frac{r}{w \rho}\right)^{\frac{2}{\eta \epsilon}}}}{\eta \epsilon w^{\frac{2}{\eta \epsilon}+1}\left(1-e^{-\pi \lambda_{b} r^{\frac{2}{\eta}}}\right)} ; \quad 0<r<(w \rho)^{\frac{1}{1-\epsilon}}
$$

Hence, the resultant intensity function after mapping and displacement can be obtained as

$$
\tilde{\lambda}(w)=\int_{0}^{\infty} \zeta(r, w) \lambda(r) \mathrm{d} r
$$

which gives (6) and proves the lemma.

Now we are in the position to give the main results of the letter, which are shown in the following theorem and corollary.

Theorem 1. The Moments of the CP for an uplink cellular network with nearest BS association, fractional path-loss inversion PC with compensation factor $\epsilon$ and activity factor $p$ 


$$
\tilde{M}_{b}=\int_{0}^{\infty} \exp \left\{-x-\frac{2 x^{1-\epsilon}}{\eta} \int_{\mathbb{1}\{\epsilon=1\}}^{\infty}\left(1-\left(\frac{y+\theta(1-p)}{y+\theta}\right)^{b}\right) y^{\frac{2}{\eta}-1} \gamma\left(1+\epsilon, x y^{\frac{2}{\eta(1-\epsilon)}}\right) \mathrm{d} y\right\} \mathrm{d} x
$$

can be approximated by $\tilde{M}_{b}$ given in (8) shown at the top of the next page, where $\mathbb{1}\{\cdot\}$ is the indicator function.

Proof: The average in (5), when conditioning on $r_{0}$, can be evaluated via the probability generating functional [10, Definition 4.3] of the PPP with the intensity function given in (6). The results are then averaged over the distribution of $r_{\circ}$ which is given by $f_{r_{\circ}}(r)=2 \pi \lambda r e^{-\pi \lambda_{b} r^{2}}, 0 \leq r \leq \infty$. The expression in (8) is then obtained by a change of variables $y=\frac{\rho w}{r^{\eta(1-\epsilon)}}$ and $x=\pi \lambda r^{2}$, followed by some mathematical manipulations. Note that for $\epsilon=1, \gamma\left(1+\epsilon, \pi \lambda_{b} x^{2} y^{\frac{2}{\eta(1-\epsilon)}}\right)=\mathbb{1}\{y>1\}$, which justifies the integration boundaries in (8).

The moments in (8) can be represented in closed form for full path-loss inversion as shown in the following corollary.

Corollary 1. The moments of the CP for an uplink cellular network with full path-loss inversion $P C$ (i.e., $\epsilon=1$ ) and activity factor $p$ can be approximated by $\tilde{M}_{b}$ given as

$$
\begin{aligned}
\tilde{M}_{b}=\exp \left\{-\frac{2}{\eta} \sum_{n=1}^{b}\left(\begin{array}{l}
b \\
n
\end{array}\right) \frac{(-1)^{n+1} p^{n} \theta^{n}}{n-\frac{2}{\eta}}\right. \\
\left.{ }_{2} F_{1}\left(n, n-\frac{2}{\eta}, n+1-\frac{2}{\eta},-\theta\right)\right\} .
\end{aligned}
$$

Proof: The proof is obtained by substituting $\epsilon=1$ in (8), applying binomial expansion, and evaluating the integral.

Interestingly, (8) and (9) show that all moments of the CP, and hence the meta distribution, are independent from the intensity of BSs. Such independence was also reported in [2] for the downlink scenario. This behavior can be justified by the fact that increasing the intensity of interfering UEs is compensated by the statistically closer intended link distances. Statistically closer intended link distances implies (i) higher received power in the fixed transmission power scenario, (ii) lower transmission powers from each interfering UE in the full channel inversion PC scenario, or both (i) and (ii) in the fractional channel inversion PC scenario. In all cases, the positive impact of closer link distances cancels the negative impact of increasing the interfering UEs for all values of $\epsilon$. Another interesting observation is that the spatially averaged CP for full channel inversion, which is obtained via $M_{1}$ in (9), is equivalent to the one derived in [7]. This reveals an interesting analogy between the interfering UEs intensity functions $\lambda(r)=\left(1-e^{-\pi \lambda_{b}\|r\|^{2}}\right) \lambda_{b}$ and $\lambda(r)=\mathbb{1}\left\{r<\left(\frac{P_{u}}{\rho}\right)^{\frac{1}{\eta}}\right\} \lambda_{b}$ that are used in [3] and [7], respectively.

Following [2, Corollary 3], replacing $b=j t$ in (8) and (9), where $j$ is the imaginary unit, an exact expression for the meta distribution can be expressed as

$$
\bar{F}(\theta, \alpha)=\frac{1}{2}+\frac{1}{\pi} \int_{0}^{\infty} \frac{\operatorname{Im}\left(e^{-t \log x} M_{j t}\right)}{t} \mathrm{~d} t,
$$

where $\operatorname{Im}(\cdot)$ denotes the imaginary part of a complex number and $M_{j t}$ is obtained by replacing $b=j t$ in (8) and (9). The expression in (10) is quite involved as it contains three nested integrals. Hence, we exploit the beta distribution approximation suggested in [2] to approximate the meta distribution of the CP in uplink cellular networks as

$$
\bar{F}(\theta, \alpha) \approx \mathcal{I}_{\alpha}\left(\frac{M_{1}\left(M_{1}-M_{2}\right)}{\left(M_{2}-M_{1}^{2}\right)}, \frac{\left(1-M_{1}\right)\left(M_{1}-M_{2}\right)}{\left(M_{2}-M_{1}^{2}\right)}\right),
$$

where $\mathcal{I}_{\alpha}(a, b)=\int_{0}^{\alpha} t^{a-1}(1-t)^{b-1} \mathrm{~d} t$ is the regularized incomplete beta function.

\section{Numerical Results}

We first validate the theoretical results via independent Monte Carlo simulations. In the simulation scenario, one PPP realization for the BSs is implemented in a $10 \times 10 \mathrm{~km}^{2}$ area. One UE is dropped in the Voronoi area of each BS. The BSs and UEs realizations are kept fixed for the entire simulation. However, the fading realization changes at each time slot and each user becomes active with probability $p$. The SIR is collected for active users only in which a wrap-around network model is implemented to avoid boundary effects. The simulation runs until 10,000 samples of SIR are collected for each UE, which are used to evaluate per UE CP. The meta distribution of the CP is then evaluated across all UEs. Unless otherwise stated, we adjust the intensity of BSs such that $\lambda_{b} p=20 \mathrm{BSs} / \mathrm{km}^{2}$ and select the network parameters as $\rho=-80 \mathrm{dBm}, \eta=4$, and $\theta=1$.

Fig. 1 plots $\bar{F}(\theta, \alpha)$ vs $\alpha$ for different values of $\epsilon$ and $p$. The close match between the simulation and analysis validates the developed model, shows the accuracy of the approximations in Lemma 2, and confirms the accuracy of the beta distribution approximation for the meta distribution for CP in the uplink. The figure also shows that decreasing the activity factor $p, \forall \epsilon$, increases the percentiles of users that achieve higher CP.

Comparing the three subfigures in Fig. 1 shows that increasing the value of $\epsilon$ increases the percentiles of users that achieves high CP (i.e., high reliability) at the expense of increasing the percentiles of users in the low coverage regime. For instance, setting $\epsilon=0$ at $p=0.5,20$ percentile of the UEs suffer from CP less than $50 \%$ and 20 percentile of the UEs enjoy a CP above $90 \%$. Adjusting $\epsilon=1$ for the same $p=0.5$, increases the UEs percentile that operates with CP above $90 \%$ to 50 at the expense of increasing the percentile of UEs operating with CP below $50 \%$ to 30 .

Fig. 2 provides more insights about the effect of $\epsilon$ and $p$ on $\bar{F}(\theta, \alpha)$. Figs. 2a and $2 \mathrm{~b}$ show that the spatially averaged $\mathrm{CP}$ (i.e., mean) is monotonically increasing, and the variance of $\mathrm{CP}$ is monotonically decreasing, when decreasing the activity factor $p$. Decreasing $p$ makes the point processes formed by the active UEs at each time slot less dependent. Hence, each active BS sees a relatively different realization of interfering UEs at each time slot. Hence, the performance of all BSs converges to the spatially average $\mathrm{CP}$ of the typical $\mathrm{BS}$, which justifies the 


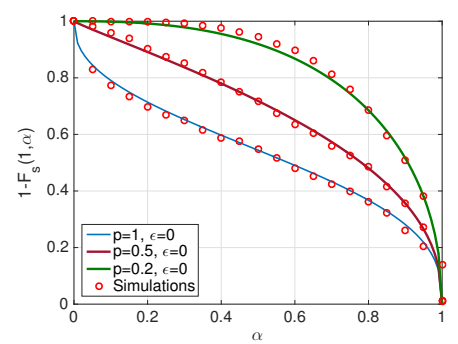

(a) $\epsilon=0$.

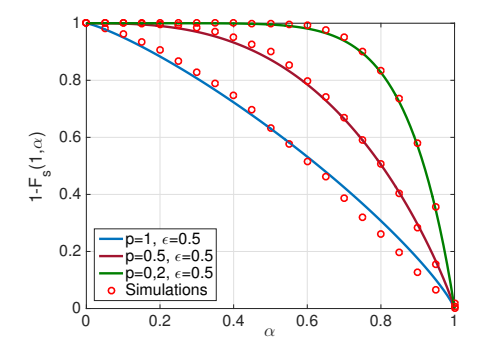

(b) $\epsilon=0.5$.

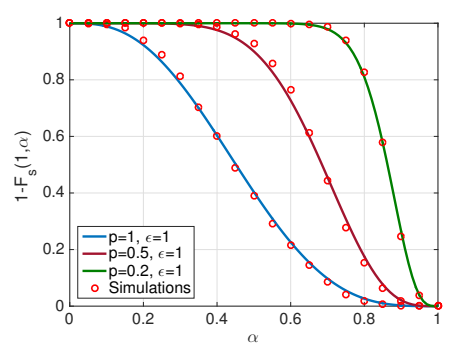

(c) $\epsilon=1$.

Fig. 1: The meta distribution for CP at different path-loss inversion factors $\epsilon$ and activity factors $p$.

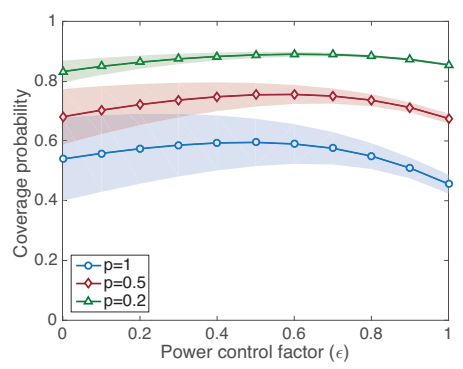

(a) $P_{s}(\theta)$ vs $\epsilon$

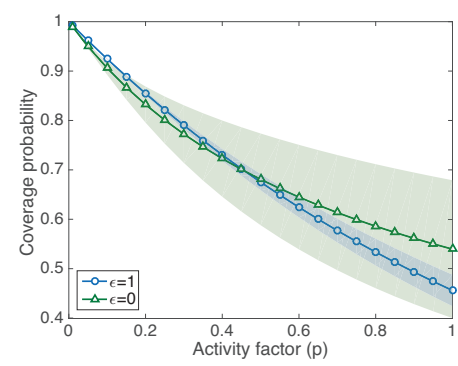

(b) $P_{s}(\theta)$ vs $p$

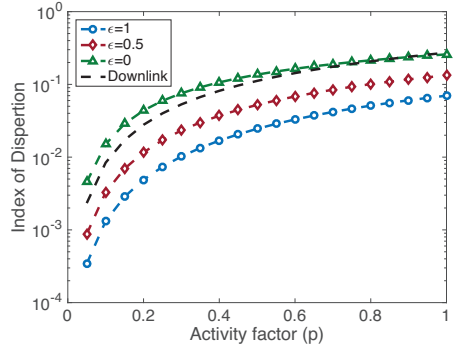

(c) IoD vs $p$

Fig. 2: Mean (solid lines), variance (shades), and index of dispersion (dotted lines) of the CP.

decrease of variance when decreasing $p$. The figures also show that, while two values of $\epsilon$ may have the same average $\mathrm{CP}$, they may have significantly different variances. For instance, for $p=0.5$, Fig. 2a shows that $\epsilon=0$ and $\epsilon=1$ have the same mean CP. However, Fig. 1 shows that going from $\epsilon=0$ to $\epsilon=1$ increases the percentile of users achieving $90 \% \mathrm{CP}$ by $30 \%$. Fig. $2 \mathrm{~b}$ manifests the significance of the PC on the variance of the $\mathrm{CP}$ despite the comparable values of mean $\mathrm{CP}$.

Since the mean $\mathrm{CP}$ varies with $p$, it is more insightful to consider the relative index of dispersion (IoD), defined as $\mathrm{IoD}=\frac{M_{2}-M_{1}^{2}}{M_{1}}$, rather than the absolute variance. Fig. $2 \mathrm{c}$ shows the IoD for the CP in uplink and downlink ${ }^{3}$ scenarios, which reveals the dispersion of the $\mathrm{CP}$ around the spatially averaged value is monotonically decreasing in $p$. Hence, decreasing the activity factor $p$ not only improves the spatially averaged $\mathrm{CP}$, but also reduces the discrepancies of the $\mathrm{CP}$ among simultaneously active users. The figure also shows comparable IoDs between the downlink and uplink without PC. Compensating for the path-loss with higher $\epsilon$ reduces the dispersion of CP among the active uplink UEs.

\section{CONCLUSIONS}

This letter studies the meta distribution of coverage probability $(\mathrm{CP})$ in uplink cellular networks with fractional path-loss inversion power control (PC). Integral forms for all moments of the $\mathrm{CP}$ are obtained for general path-loss compensation factor $\epsilon$, which reduce to closed form expressions for full-path-loss inversion. The moments are used to write an exact integral expressions for the meta distribution and for moment matching approximation for the meta distribution via the beta distribution. To this end, the effects of PC and users activity $(p)$ on the CP

\footnotetext{
${ }^{3}$ The CP moments for the downlink are obtained from [2, Theorem 3].
}

percentiles for uplink users are discussed. The results show that two values of $\epsilon$ that show the same spatially averaged CP may hide significant discrepancies in the $\mathrm{CP}$ variances, which manifests the importance of the meta distribution. The results also show that the discrepancies among the CPs monotonically decreases in $p$ and/or $\epsilon$.

\section{REFERENCES}

[1] H. ElSawy, A. Sultan-Salem, M. S. Alouini, and M. Z. Win, "Modeling and analysis of cellular networks using stochastic geometry: A tutorial," IEEE Commun. Surveys Tuts., vol. 19, no. 1, pp. 167-203, Firstquarter 2017.

[2] M. Haenggi, "The meta distribution of the SIR in Poisson bipolar and cellular networks," IEEE Trans. Wireless Commun., vol. 15, no. 4, pp. 2577-2589, Apr. 2016.

[3] S. Singh, X. Zhang, and J. G. Andrews, "Joint rate and SINR coverage analysis for decoupled uplink-downlink biased cell associations in HetNets," IEEE Trans. Wireless Commun., vol. 14, no. 10, pp. 5360-5373, Oct. 2015.

[4] M. D. Renzo and P. Guan, "Stochastic geometry modeling and systemlevel analysis of uplink heterogeneous cellular networks with multiantenna base stations," IEEE Trans. Commun., vol. 64, no. 6, pp. 24532476, Jun. 2016.

[5] R. K. Ganti and J. G. Andrews, "Correlation of link outages in lowmobility spatial wireless networks," in 2010 Conference Record of the Forty Fourth Asilomar Conference on Signals, Systems and Computers, Nov. 2010, pp. 312-316.

[6] 3GPP TS 36.213, LTE-Evolved Universal Terrestrial Radio Access (EUTRA), Physical Layer Procedures. Release 8, 2009.

[7] H. ElSawy and E. Hossain, "On stochastic geometry modeling of cellular uplink transmission with truncated channel inversion power control," IEEE Trans. Wireless Commun., vol. 13, no. 8, pp. 4454-4469, Aug. 2014.

[8] F. J. Martin-Vega, G. Gomez, M. C. Aguayo-Torres, and M. D. Renzo, "Analytical modeling of interference aware power control for the uplink of heterogeneous cellular networks," IEEE Trans. Wireless Commun., vol. 15, no. 10, pp. 6742-6757, Oct. 2016.

[9] M. Haenggi, "User point processes in cellular networks," IEEE Wireless Commun. Lett., to appear.

[10] - Stochastic Geometry for Wireless Networks. Cambridge University Press, 2012. 